\title{
Research on Evaluation Strategy of University Library Database
}

\author{
$\mathrm{Xu}$ Wang \\ Tianjin Maritime College, Tianjin, 300350
}

\begin{abstract}
With the continuous development of network technology and computer storage technology, the library resource structure has also undergone profound changes. Electronic resources, as an important part of the library literature resources, has been developing rapidly in recent years, and a considerable number of university library database funds have accounted for one-third of the total funding of literature resources. How to understand the service effect of the database more accurately, establish the scientific and reasonable collection of literature resources system, improve the performance of funds investment and how to choose high-quality database resources are major issues we face.
\end{abstract}

Keywords: evaluation strategy, university, library, database

\section{Introduction}

The high purchase cost of the database requires the purchasing decision-maker to have sufficient readers to use the research foundation before purchasing, and also to have a large number of statistical evaluations after purchase to provide the scientific basis for the renewal. Whether to purchase new database resources, or to renew or delete existing e-journal collections, we are faced with a new problem: how to better understand the service effectiveness of the database, how to establish a scientific and reasonable collection of resources system, Investment performance. At present, many of our university library database usage and procurement model is not optimistic. Therefore, how to ensure that the library with limited funds to purchase to facilitate the development of the subject database resources has become the current university library sector to be resolved 
a major problem. With the increasing number of electronic resources, university libraries in the acquisition of electronic resources and the use of funds have also been increasing.

\section{The Meaning of Database Evaluation}

The use of electronic resources or database evaluation is the use of electronic resources in the process of displaying a variety of properties and the use of the effect of detection, the use of electronic resources to determine the value and feedback of various information, so as to develop new electronic resources Construction objectives and programs to control the development of electronic resources to provide an objective basis for the activities. From the macroscopic point of view, the use of electronic resources evaluation is the overall electronic resources of the library system status, function, value and its role in monitoring and evaluation; from a microscopic point of view, the library of electronic resources evaluation is a specific type of Electronic resources, the value of the use of the results, availability, etc. to evaluate, in which a specific type of electronic resources refers to a type of database, or electronic journals, or e-books. Evaluation of the use of electronic resources belong to the scope of postevaluation, that is, relative to the pre-procurement of resources before the assessment conducted by the evaluation of the use of resources.

\section{The Existing Problems of Database Selection}

\subsection{Limited library funding}

Each year, the library's library resources are limited, and the price of the database is increasing every year. Some libraries have difficulties in maintaining the original database renewal, not to mention the purchase of new databases.

\subsection{The feedback of the purchase information is difficult}

A database in the trial process, we will ask the database provider to provide readers of the school usage statistics. In general, if the number of visits to the database, then the database of resources that readers are interested in is the reader needs, and thus can consider (rather than decide) the official purchase. However, because the data provided by the database provider is generally EXCEL format, they may modify or create data, increase the utilization rate to sell their products, which we have no way to confirm. Therefore, we need to use the database for readers to investigate, combined with the data provided by the database to understand the real use, as the basis for decision-making. But many readers focus on the use of the process do not attach importance to communication and library procurement staff. Library procurement staff and readers of the communication 
between the disjoint, making the database query the views of the purchase there is a great difficulty.

\subsection{The cooperation between departments is not harmonious}

The purchase of the database involves departments such as the Department of Interviews, Periodicals, Technology, and Information. The Department of Interviews is the department that involves the introduction of the database. The periodicals are involved in the bundling of the periodicals. The full-text electronic periodicals generally require the purchasers to order a considerable quantity of the paper. Information Department involved in the use of the database, training and other issues, the technology involved in the mirror database installation, update and maintenance issues. The efficiency of the division of labor and cooperation in the procurement of electronic resources has a direct impact on the use of electronic resources by readers.

\section{The Principle of Database Selection}

The principle of procurement of database resources is closely related to the disciplines and readership of library construction. It is necessary to adhere to the principle of serving users, improving resource utilization and paying attention to information feedback as the principle of procurement. While maintaining traditional library information service functions, Information technology provides teachers and students with access to global information resources. In order to avoid the blindness caused by the museum's financial and human waste, should follow the principles of systemic, pertinence, coordination and characteristics, authority, selectivity, economy, legitimacy:

\subsection{Systematic principles}

Similar to the procurement of paper-based documents, the selection of the database should be consistent with the general principles of the library collection, in line with the museum's collection features, the reader object, and print the document resources into a whole to form a complete meet the needs of readers at all levels Document Information Resource Guarantee System. Systematic principles require procurement personnel to pay attention to the following three points: First, in the collection of funds budget, to determine the choice of various electronic publications and procurement ratio, the proportion of established electronic collection structure, the subject, language, time and region composition of the implementation of such collections. Attention should be paid to the system integrity of the contents and formal structure of the electronic collection system so that the electronic collection system with the best structure and function can be formed after long-term accumulation. Second, the focus and characteristics Electronic collections in the content, quantity, variety to maintain a certain degree of systemic and integrity, a variety of major search tools and full text database to 
be included intact. Third, the procurement of electronic resources should be planned, including the long-term development plans and short-term procurement plan, procurement of documents to be timely.

\subsection{The principle of relevance}

To strengthen the user needs research, the establishment of key service disciplines, the characteristics of the user groups and needs of the decision to collect content, the construction of subject database and network resource navigation system. In the collection of paid electronic literature, should try to refine the choice of efforts to reduce the zero utilization of electronic literature order. Currently, large-scale integrated digital resources are still in the buyer's market, for example, in the choice of e-books, the majority of e-book suppliers do not support the library according to the name of the directory catalog sub-selected, only by the supplier to the category, Rough selection, resulting in the library to buy some of the books they do not need.

\subsection{The principle of coordination and characterization}

Database and paper literature is the library of the two most important documents, and there must be cross between the two and repeat the collection must pay attention to the coordination of the two, otherwise it will result in waste of funds and collection of blind spots. In addition, in the era of resource sharing, there is no characteristic of resources to lose the sharing of the necessary, therefore, in the collection of the database and we should pay attention to screening, in order to gradually form the museum's collection characteristics.

\section{The University Library Database Evaluation Index}

The total amount of data in measuring the size and level of an information service organization, the role of the total amount of information resources is self-evident. Characteristic databases are no exception. This is an objective indicator, the higher the value of the indicator, the higher the level of the database being evaluated. In the actual evaluation, you can examine the total number of system resources records (in million units), you can also examine the storage capacity of resources (in MB). Data quality mainly from two aspects, one is whether the text typos and the second is the degree of clarity of the scanned image and whether there is significant tilt and so on.

\subsection{Searching system and its functions}

Mainly refers to the database and retrieval platform related to the basic information and use of the system used to evaluate the various aspects of the use of the search function. The retrieval system provided by the database is one of the most important indicators of a database, but it is also one of the most complex 
and difficult to evaluate because different users often feel different about the retrieval system, and the retrieval system Functional evaluation is difficult to use quantitative indicators to analyze.

\subsection{The use of the database}

The use of the database can be used to reflect the comparison of quantitative and objective indicators, and the indicators can also be constantly using a certain period of time to evaluate the data, and can be dynamically evaluated, so the evaluation of the index is particularly important. The usage index can include the number of logins, the number of searches, the number of full text downloads, the number of non-normal use, the number of normal use, the reader satisfaction.

\subsection{Cost accounting}

The economy of the database is used as an evaluation index, which is mainly caused by the limitation of the library of our country. In the digitalized and networked environment, the cost of construction and maintenance of the database is quite high and requires a lot of capital, equipment and manpower. Therefore, it is necessary to evaluate the input cost and benefit of the digital collections of library to promote the sound development of library digital library construction.

\section{Conclusion}

The construction of the database is especially important in the library. The good evaluation index is helpful to guide the construction of the characteristic database system, standardize the selection and index of the resources, unify the technical standards in construction, enhance the interoperability and guarantee the quality of the characteristic database construction, build a foundation for the sharing of resources.

\section{References}

[1] Sun Fang, Zhao Liangying. Research on Evaluation Indexes of University Library Database. Library Work and Study, 55(12), pp.68-70, 2011

[2] Li Mingwu, Lv Xianjing. Analysis of Evaluation Indexes of Characteristic Database System Construction. Modern Information, 8 (5), pp.87- 91, 20013

[3] Wang Xuelian, Liu Qinghua. Foreign electronic journals access and use of assessment. Library Science, 12(10), pp. 18- 20, 2012

[4] Yang Zuguo, Zhu Guiling .Comparison of foreign full-text electronic journals database. Journal of Information Science, 9(6), pp.58- 61, 2011

[5] Xiao Long, Zhang Yuhong. Establishment of the Evaluation Index System of Electronic Resources. Journal of Academic Libraries, 8(4), pp.85-87, 2014 